\title{
Analytic Kramer kernels, Lagrange-type interpolation series and de Branges spaces
}

\author{
Antonio G. García ${ }^{a *}$, Miguel A. Hernández-Medina ${ }^{b}$ and Franciszek \\ Hugon Szafraniec ${ }^{c}$ \\ ${ }^{a}$ Departamento de Matemáticas, Universidad Carlos III de Madrid, \\ Avda. de la Universidad 30, Leganés-Madrid 28911, Spain; \\ ${ }^{b}$ Departamento de Matemática Aplicada, E.T.S.I.T., U.P.M., Avda. Complutense s/n, \\ Madrid 28040, Spain; ${ }^{c}$ Instytut Matematyki, Uniwersytet Jagielloński, ul. Lojasiewicza 6, \\ 30348 Kraków, Poland \\ Communicated by W. Koepf
}

(Received 12 November 2010; final version received 9 December 2010)

\begin{abstract}
The classical Kramer sampling theorem provides a method for obtaining orthogonal sampling formulas. In particular, when the involved kernel is analytic in the sampling parameter it can be stated in an abstract setting of reproducing kernel Hilbert spaces of entire functions which includes as a particular case the classical Shannon sampling theory. This abstract setting allows us to obtain a sort of converse result and to characterize when the sampling formula associated with an analytic Kramer kernel can be expressed as a Lagrange-type interpolation series. On the other hand, the de Branges spaces of entire functions satisfy orthogonal sampling formulas which can be written as Lagrange-type interpolation series. In this work some links between all these ideas are established.
\end{abstract}

Keywords: analytic Kramer kernels; Lagrange-type interpolation series; de Branges spaces

AMS Subject Classifications: 46E22; 42C15;94A20

\section{Statement of the problem}

The classical Kramer sampling theorem provides a method for obtaining orthogonal sampling theorems $[1,2,3]$. The statement of this general result is as follows. Let $K$ be a complex function defined on $D \times I$, where $I \subset \mathbb{R}$ is an interval and $D$ is an open subset of $\mathbb{R}$, and such that for every $t \in D$ the sections $K(\cdot, t)$ are in $\mathcal{L}^{2}(I)$. Assume that there exists a sequence of distinct real numbers $\left\{t_{n}\right\} \subset D$, indexed by integers, such that $\left\{K\left(x, t_{n}\right)\right\}$ is a complete orthogonal sequence of functions for $\mathcal{L}^{2}(I)$.

*Corresponding author. Email: agarcia@math.uc3m.es 
Then for any $F$ of the form

$$
F(t)=\int_{I} f(x) K(x, t) \mathrm{d} x, \quad t \in D,
$$

where $f \in \mathcal{L}^{2}(I)$, we have

$$
F(t)=\lim _{N \rightarrow \infty} \sum_{|n| \leq N} F\left(t_{n}\right) S_{n}(t)
$$

with

$$
S_{n}(t):=\frac{\int_{I} K(x, t) \overline{K\left(x, t_{n}\right)} \mathrm{d} x}{\int_{I}\left|K\left(x, t_{n}\right)\right|^{2} \mathrm{~d} x} .
$$

The series in (2) converges absolutely and uniformly on subsets of $D$ where $\|K(\cdot, t)\|_{\mathcal{L}^{2}(I)}$ is bounded.

The Kramer theorem has played a very significant role in sampling theory, interpolation theory, signal analysis and so forth (see the survey articles [4,5]). Besides, it has been the cornerstone for a significant amount of mathematical literature on the topic of sampling theorems associated with differential or difference problems which has flourished for the past few years. As for a small but significant selection of examples see, for instance $[1,6,3]$ and references therein.

If $D$ is an open subset of $\mathbb{C}$, which is our assumption from now on, a possibility of considering kernels analytic in the sampling parameter $t$ appears (see [7] for implementation of this idea). If $D$ is just the whole of $\mathbb{C}$, any function $F$ defined by (1) is an entire function, as are all the sampling functions (3). This gives rise to the consideration of analytic Kramer kernels; for a complete meaning see Definition 2.2.

The abstract version of the analytic Kramer sampling theorem consists in replacing the space $\mathcal{L}^{2}(I)$ by an arbitrary separable complex Hilbert space, say $\mathcal{H}$. A given $\mathcal{H}$-valued function $K$ defined on $\mathbb{C}$, for each $x \in \mathcal{H}$, which plays a role of the Kramer kernel, gives rise to a reproducing kernel Hilbert space (RKHS in short) $\mathcal{H}_{K}$ within which the sampling is performed. A little bit more, ${ }^{1}$ assuming the existence of two sequences $\left\{z_{n}\right\}_{n=1}^{\infty}$ in $\mathbb{C}$ and $\left\{a_{n}\right\}_{n=1}^{\infty}$ in $\mathbb{C} \backslash\{0\}$ such that $K\left(z_{n}\right)=a_{n} x_{n}$ for each $n \in \mathbb{N}$, the analytic Kramer theorem in its abstract version says there is a sequence $\left\{S_{n}\right\}_{n=1}^{\infty} \subset \mathcal{H}_{K}$ of sampling functions such that

$$
f(z)=\sum_{n=1}^{\infty} f\left(z_{n}\right) \frac{S_{n}(z)}{a_{n}}, \quad z \in \mathbb{C} .
$$

Regarding formula (4) some challenging problems arise. The first one is to decide when a pointwise convergent sampling formula in $\mathcal{H}_{K}$ as (4) comes out from a Kramer kernel (Section 2.3). The second one is to give a necessary and sufficient condition to ensure that the corresponding sampling formula can be written as a Lagrange-type interpolation series. The latter is not always true as a counterexample in Section 2.5 will show. Roughly speaking, the aforesaid necessary and sufficient condition concerns the stability of functions to belong to the space $\mathcal{H}_{K}$ on removing a finite number of zeros (see Section $2.5 \mathrm{infra}$ ). The third one is to decide when a space $\mathcal{H}_{K}$ is a de Branges one (see Section 3 for details on these spaces). These spaces can be seen as particular cases of $\mathcal{H}_{K}$ spaces where orthogonal sampling formulas exist that 
can be expressed as Lagrange-type interpolation series. All these steps will be carried out throughout the remaining sections.

\section{Analytic Kramer sampling theory within the space $\mathcal{H}_{K}$}

Throughout this article $\langle\cdot,-\rangle$ followed by a subscript indicating the Hilbert space in question stands always for an inner product.

\subsection{More on the space $\mathcal{H}_{K}$}

Suppose we are given a separable complex Hilbert space and an abstract kernel $K$ which is nothing but a $\mathcal{H}$-valued function on $\mathbb{C}$. Set $f_{x}(z):=\langle K(z), x\rangle_{\mathcal{H}}$ and denote by $\mathcal{H}_{K}$ the collection of all such functions $f_{x}, x \in \mathcal{H}$. It is an RKHS coming from the transforms $^{2} K(z), z \in \mathbb{C}$, and corresponding to the kernel $(z, w) \mapsto\langle K(z), K(w)\rangle_{\mathcal{H}}$. Note that the mapping $\mathcal{T}$ given by

$$
\mathcal{H} \ni x \stackrel{\mathcal{T}}{\longrightarrow} f_{x} \in \mathcal{H}_{K}
$$

is an antilinear mapping from $\mathcal{H}$ onto $\mathcal{H}_{K}$. It is injective if and only if the set $\{K(z)\}_{z \in \mathbb{C}}$ is complete in $\mathcal{H}$. In particular, if there exists a sequence $\left\{z_{n}\right\}_{n=1}^{\infty}$ in $\mathbb{C}$ such that $\left\{K\left(z_{n}\right)\right\}_{n=1}^{\infty}$ is a Riesz basis for $\mathcal{H}$, then $\mathcal{T}$ is an anti-linear isometry from $\mathcal{H}$ onto $\mathcal{H}_{K}$. Recall that a Riesz basis in a separable Hilbert space $\mathcal{H}$ is the image of an orthonormal basis by means of a boundedly invertible operator. Any Riesz basis $\left\{x_{n}\right\}_{n=1}^{\infty}$ has a unique biorthonormal (dual) Riesz basis $\left\{y_{n}\right\}_{n=1}^{\infty}$, i.e. $\left\langle x_{n}, y_{m}\right\rangle_{\mathcal{H}}=\delta_{n, m}$, such that the expansions

$$
x=\sum_{n=1}^{\infty}\left\langle x, y_{n}\right\rangle_{\mathcal{H}} x_{n}=\sum_{n=1}^{\infty}\left\langle x, x_{n}\right\rangle_{\mathcal{H}} y_{n}
$$

hold for every $x \in \mathcal{H}$ (see [8] for more details and proofs).

The convergence in the norm $\|\cdot\|_{\mathcal{H}_{K}}$ implies pointwise convergence which is uniform on those subsets of $\mathbb{C}$ where the function $z \mapsto\|K(z)\|_{\mathcal{H}}$ is bounded.

Like in the classical case the following result holds: $\mathcal{H}_{K}$ is an RKHS of entire functions if and only if the kernel $K$ is analytic in $\mathbb{C}[9$, p. 266]. Another characterization of the analyticity of the functions in $\mathcal{H}_{K}$ is given in terms of Riesz bases. Suppose that a Riesz basis $\left\{x_{n}\right\}_{n=1}^{\infty}$ for $\mathcal{H}$ is given and let $\left\{y_{n}\right\}_{n=1}^{\infty}$ be its dual Riesz basis; expanding $K(z)$, where $z \in \mathbb{C}$ is fixed in this basis we obtain

$$
K(z)=\sum_{n=1}^{\infty}\left\langle K(z), y_{n}\right\rangle_{\mathcal{H}} x_{n},
$$

where the coefficients $\left\langle K(z), y_{n}\right\rangle_{\mathcal{H}}$ as functions in $z$ are in $\mathcal{H}_{K}$. The following result holds.

Lemma 2.1 Let $\left\{x_{n}\right\}_{n=1}^{\infty}$ and $\left\{y_{n}\right\}_{n=1}^{\infty}$ be a pair of dual Riesz bases for $\mathcal{H}$. Then, $\mathcal{H}_{K}$ is an RKHS of entire functions if and only if all the functions

$$
S_{n}(z):=\left\langle K(z), y_{n}\right\rangle_{\mathcal{H}}, \quad z \in \mathbb{C}
$$

are entire and $\|K(\cdot)\|_{\mathcal{H}}$ is bounded on compact sets of $\mathbb{C}$. 
Proof The necessary condition is obvious. The sufficient condition is a straightforward consequence of Montel's theorem. Indeed, $K$ will be analytic in $\mathbb{C}$ if and only if the function $f(z):=\langle K(z), x\rangle_{\mathcal{H}}$ is analytic in $\mathbb{C}$ for each $x \in \mathcal{H}$. By using the continuity of the inner product we get

$$
f(z)=\langle K(z), x\rangle_{\mathcal{H}}=\left\langle\sum_{n=1}^{\infty} S_{n}(z) x_{n}, x\right\rangle_{\mathcal{H}}=\sum_{n=1}^{\infty} S_{n}(z)\left\langle x_{n}, x\right\rangle_{\mathcal{H}} .
$$

Applying the Cauchy-Schwarz inequality we get

$$
\left|\sum_{n=1}^{N} S_{n}(z)\left\langle x_{n}, x\right\rangle_{\mathcal{H}}\right|^{2} \leq\left(\sum_{n=1}^{N}\left|S_{n}(z)\right|^{2}\right)\left(\sum_{n=1}^{N}\left|\left\langle x_{n}, x\right\rangle_{\mathcal{H}}\right|^{2}\right) \leq \sum_{n=1}^{\infty}\left|S_{n}(z)\right|^{2}\|x\|^{2} .
$$

The Riesz basis condition implies that there exist two constants $0<A \leq B$ such that $A\|K(z)\|_{\mathcal{H}}^{2} \leq \sum_{n=1}^{\infty}\left|S_{n}(z)\right|^{2} \leq B\|K(z)\|_{\mathcal{H}}^{2}$. As a consequence, the partial sums in the series of (7) are uniformly bounded on compact sets of $\mathbb{C}$. Consequently, Montel's theorem assures the existence of a subsequence which will converge to an entire function which necessarily coincides with $f$. Hence, $f$ is an entire function.

\subsection{Sampling in the space $\mathcal{H}_{K}$}

Consider the data

$$
\left\{z_{n}\right\}_{n=1}^{\infty} \in \mathbb{C} \quad \text { and } \quad\left\{a_{n}\right\}_{n=1}^{\infty} \in \mathbb{C} \backslash\{0\} .
$$

Definition 2.2 An analytic kernel $K: \mathbb{C} \rightarrow \mathcal{H}$ is said to be an analytic Kramer kernel (with respect to the data (8)) if it satisfies $K\left(z_{n}\right)=a_{n} x_{n}, n \in \mathbb{N}$, for some Riesz basis $\left\{x_{n}\right\}_{n=1}^{\infty}$ of $\mathcal{H}$. A sequence $\left\{S_{n}\right\}_{n=1}^{\infty}$ of functions in $\mathcal{H}_{K}$ is said to be have the interpolation property (with respect to the data (8)) if

$$
S_{n}\left(z_{m}\right)=a_{n} \delta_{n, m} .
$$

An analytic kernel is an analytic Kramer one if and only if the sequence of functions $\left\{S_{n}\right\}_{n=1}^{\infty}$ in $\mathcal{H}_{K}$ given by (6), where $\left\{y_{n}\right\}_{n=1}^{\infty}$ is the dual Riesz basis of $\left\{x_{n}\right\}_{n=1}^{\infty}$, has the interpolation property with respect to the same data (8).

Under the notation introduced so far the following abstract version of the classical Kramer sampling theorem sampling [2] holds (see also [10]):

THEOREM 2.3 Let $K: \mathbb{C} \rightarrow \mathcal{H}$ be an analytic Kramer kernel, and assume that the interpolation property (9) holds for some sequences $\left\{z_{n}\right\}_{n=1}^{\infty}$ in $\mathbb{C}$ and $\left\{a_{n}\right\}_{n=1}^{\infty}$ in $\mathbb{C} \backslash\{0\}$. Let $\mathcal{H}_{K}$ be the corresponding $R K H S$ of entire functions. Then any $f \in \mathcal{H}_{K}$ can be recovered from its samples $\left\{f\left(z_{n}\right)\right\}_{n=1}^{\infty}$ by means of the sampling series

$$
f(z)=\sum_{n=1}^{\infty} f\left(z_{n}\right) \frac{S_{n}(z)}{a_{n}}, \quad z \in \mathbb{C} .
$$

This series converges absolutely and uniformly on compact subsets of $\mathbb{C}$.

Proof First notice that $\lim _{n \rightarrow \infty}\left|z_{n}\right|=+\infty$; otherwise the sequence $\left\{z_{n}\right\}_{n=1}^{\infty}$ contains a bounded subsequence and hence, the entire function $S_{n} \equiv 0$ for all $n \in \mathbb{N}$ which 
contradicts (9). The anti-linear mapping $\mathcal{T}$ given by (5) is a bijective isometry between $\mathcal{H}$ and $\mathcal{H}_{K}$. As a consequence, the functions $\left\{S_{n}=\mathcal{T}\left(y_{n}\right)\right\}_{n=1}^{\infty}$ form a Riesz basis for $\mathcal{H}_{K}$; let $\left\{T_{n}\right\}_{n=1}^{\infty}$ be its dual Riesz basis. Expanding any $f \in \mathcal{H}_{K}$ in this basis we obtain

$$
f(z)=\sum_{n=1}^{\infty}\left\langle f, T_{n}\right\rangle_{\mathcal{H}_{K}} S_{n}(z)
$$

Moreover,

$$
\left\langle f, T_{n}\right\rangle_{\mathcal{H}_{K}}={\overline{\left\langle x, x_{n}\right\rangle_{\mathcal{H}}}}=\left\langle\frac{K\left(z_{n}\right)}{a_{n}}, x\right\rangle_{\mathcal{H}}=\frac{f\left(z_{n}\right)}{a_{n}} .
$$

Since a Riesz basis is an unconditional basis, the sampling series will be pointwise unconditionally convergent and hence, absolutely convergent. The uniform convergence is a standard result in the setting of the RKHS theory since $z \mapsto\|K(z)\|_{\mathcal{H}}$ is bounded on compact subsets of $\mathbb{C}$.

Riesz bases theory assures the existence of two constants $0<A \leq B$ such that

$$
A\|f\|_{\mathcal{H}_{K}}^{2} \leq \sum_{n=1}^{\infty}\left|f\left(z_{n}\right) / a_{n}\right|^{2} \leq B\|f\|_{\mathcal{H}_{K}}^{2} \quad \text { for all } f \in \mathcal{H}_{K} .
$$

In the case that the Riesz basis $\left\{x_{n}\right\}_{n=1}^{\infty}$ is an orthonormal one we say that (10) is an orthogonal sampling formula.

\subsection{A converse result}

An interesting converse problem is to decide when a sampling formula as (10), pointwise convergent in $\mathcal{H}_{K}$, implies the Kramer kernel condition for $K$. From formula (10) in Theorem 2.3 we derive that

- the sequence $\left\{f\left(z_{n}\right) / a_{n}\right\}_{n=1}^{\infty}$ belongs to $\ell^{2}(\mathbb{N})$ for any $f \in \mathcal{H}_{K}$, and

- $\sum_{n=1}^{\infty} \alpha_{n} S_{n}(z)=0$ for all $z \in \mathbb{C}$ and $\left\{\alpha_{n}\right\}_{n=1}^{\infty} \in \ell^{2}(\mathbb{N})$ implies that $\alpha_{n}=0$ for all $n \in \mathbb{N}$, due to the uniqueness of a Riesz basis expansion in the RKHS $\mathcal{H}_{K}$.

It is worth pointing out that these two conditions are also sufficient to prove that $K$ is an (analytic) Kramer kernel. For a proof see [11] under minor changes.

\subsection{Existence of analytic Kramer kernels}

As it was proved in [12], associated with an arbitrary sequence of complex numbers $\left\{z_{n}\right\}_{n=1}^{\infty}$ such that $\lim _{n \rightarrow \infty}\left|z_{n}\right|=+\infty$, there exists an analytic Kramer kernel. Indeed, given the sequence $\left\{z_{n}\right\}_{n=1}^{\infty}$, consider a sequence $\left\{a_{n}\right\}_{n=1}^{\infty}$ in $\mathbb{C} \backslash\{0\}$ such that $\sum_{z_{n} \neq 0}\left|a_{n} / z_{n}\right|^{2}<\infty$ (set $a_{k}=1$ in the case $z_{k}=0$ ). Now, let $P$ be an entire function having only simple zeros at $\left\{z_{n}\right\}_{n=1}^{\infty}$; this is allowed by the theorem of Weierstrass $\left[8\right.$, p. 54]. In a separable Hilbert space $\mathcal{H}$ with dual Riesz bases $\left\{x_{n}\right\}_{n=1}^{\infty}$ and $\left\{y_{n}\right\}_{n=1}^{\infty}$ 
define the mapping $K: \mathbb{C} \rightarrow \mathcal{H}$ as:

$$
K(z):=\sum_{n=1}^{\infty} \frac{a_{n} P(z)}{z-z_{n}} x_{n} \in \mathcal{H},
$$

where the convergence of the series is in the norm of $\mathcal{H}$. Then $K$ defines an analytic Kramer kernel and the sampling formula for each $f$ in the corresponding RKHS $\mathcal{H}_{K}$ reads as

$$
f(z)=\sum_{n=1}^{\infty} f\left(z_{n}\right) \frac{P(z)}{\left(z-z_{n}\right) P^{\prime}\left(z_{n}\right)}, \quad z \in \mathbb{C} .
$$

The entire functions $S_{n}(z)=a_{n} P(z) /\left(z-z_{n}\right)=\left\langle K(z), y_{n}\right\rangle_{\mathcal{H}}, n \in \mathbb{N}$, satisfy the interpolation condition $S_{n}\left(z_{m}\right)=a_{m} P^{\prime}\left(z_{m}\right) \delta_{n, m}$. In addition, the function $z \mapsto\|K(z)\|_{\mathcal{H}}$ is uniformly bounded on compact subsets of $\mathbb{C}$. The Riesz basis condition on $\left\{y_{n}\right\}_{n=1}^{\infty}$ gives the existence of a constant $A$ such that

$$
\|K(z)\|_{\mathcal{H}}^{2} \leq A \sum_{n=1}^{\infty}\left|\frac{a_{n} P(z)}{z-z_{n}}\right|^{2}, \quad z \in \mathbb{C},
$$

and the series is uniformly bounded on compact subsets [12]. Thus, $K$ defines an analytic Kramer kernel. As a consequence, Theorem 2.3 assures that any function $f \in \mathcal{H}_{K}$ can be recovered from its samples $\left\{f\left(z_{n}\right)\right\}_{n=1}^{\infty}$ by means of the Lagrange-type interpolation formula

$$
f(z)=\sum_{n=1}^{\infty} f\left(z_{n}\right) \frac{a_{n} P(z) /\left(z-z_{n}\right)}{a_{n} P^{\prime}\left(z_{n}\right)}=\sum_{n=1}^{\infty} f\left(z_{n}\right) \frac{P(z)}{\left(z-z_{n}\right) P^{\prime}\left(z_{n}\right)}, \quad z \in \mathbb{C} .
$$

\subsection{Lagrange-type interpolation series}

A more difficult question concerns whether the sampling expansion (10) can be written, in general, as a Lagrange-type interpolation series as in (12). As it was proved in [12] for the case where $\left\{x_{n}\right\}_{n=1}^{\infty}$ in Definition 2.2 is an orthonormal basis for $\mathcal{H}$, a necessary and suficient condition involves the following algebraic property.

Definition 2.4 A space $\mathcal{H}$ of entire functions has the zero-removing property (ZR property hereafter) if for any function $g \in \mathcal{H}$ and any zero $w$ of $g$ the function $g(z) /(z-w)$ belongs to $\mathcal{H}$.

The Paley-Wiener space $P W_{\pi \sigma}:=\left\{f \in \mathcal{L}^{2}(\mathbb{R}) \cap C(\mathbb{R})\right.$, supp $\left.\widehat{f} \subseteq[-\pi \sigma, \pi \sigma]\right\}$, where $\widehat{f}$ stands for the Fourier transform of $f$, clearly satisfies the ZR property. Indeed, the Whittaker-Shannon-Kotel'nikov sampling formula for $f \in P W_{\pi \sigma}$ (cf. [3, p. 16]),

$$
f(z)=\sum_{n=-\infty}^{\infty} f\left(\frac{n}{\sigma}\right) \frac{\sin \pi(\sigma z-n)}{\pi(\sigma z-n)}, \quad z \in \mathbb{C},
$$

can be written as a Lagrange-type interpolation series by taking $P(z)=(\sin \pi \sigma z) / \pi$. Without using Theorem 2.5, the ZR property follows from the characterization of $P W_{\pi \sigma}$ which uses the classical Paley-Wiener Theorem [8, p. 101], i.e. $P W_{\pi \sigma}=\left\{f \in \mathcal{H}(\mathbb{C}):|f(z)| \leq A \mathrm{e}^{\pi \sigma|z|},\left.f\right|_{\mathbb{R}} \in \mathcal{L}^{2}(\mathbb{R})\right\}$. 
On the other hand, it is easy to construct analytic Kramer kernels $K$ such that the corresponding spaces $\mathcal{H}_{K}$ do not satisfy the ZR property. Indeed, let $K: \mathbb{C} \rightarrow \mathcal{L}^{2}[-\pi, \pi]$ be the kernel defined by $[K(z)](x):=\frac{1}{\sqrt{2 \pi}} \mathrm{e}^{\mathrm{i} z^{2} x}$. It is an analytic Kramer kernel and its Taylor series around $z=0$ is given by $[K(z)](x)=$ $\sum_{k=0}^{\infty}(\mathrm{i} x)^{k} z^{2 k} / k$ !. Hence, the Taylor series of any function $f(z)=\langle K(z), F\rangle_{\mathcal{L}^{2}[-\pi, \pi]}$ in $\mathcal{H}_{K}$, where $F \in \mathcal{L}^{2}[-\pi, \pi]$ is of the form

$$
f(z)=\sum_{k=0}^{\infty} \frac{c_{k}}{k !} z^{2 k}, \quad z \in \mathbb{C},
$$

where $c_{k}=\left\langle(\mathrm{i} x)^{k}, F\right\rangle$, i.e. $f$ is an even function. Let $G$ be a nonzero function in $\mathcal{L}^{2}[-\pi, \pi]$ such that $\int_{-\pi}^{\pi} G=0$. The function $G$ is orthogonal to $K(0)$, and consequently, the entire function $g(z)=\langle K(z), G\rangle_{\mathcal{L}^{2}[-\pi, \pi]}$ verifies $g(0)=0$. We have

$$
\frac{g(z)}{z}=\sum_{k=0}^{\infty} \frac{c_{k}}{k !} z^{2 k-1}, \quad \text { where } c_{k}=\left\langle(\mathrm{i} x)^{k}, G\right\rangle .
$$

Clearly, the entire function $g(z) / z$ does not belong to $\mathcal{H}_{K}$ and therefore, the space $\mathcal{H}_{K}$ does not satisfy the ZR property.

For the case of analytic Kramer kernels $K$ one can find a necessary and sufficient condition assuring when the corresponding spaces $\mathcal{H}_{K}$ satisfy the ZR property.

THEOREM 2.5 Let $\mathcal{H}_{K}$ be an RKHS of entire function obtained from an analytic Kramer kernel $K$ with respect to the sequence $\left\{z_{n}\right\}_{n=1}^{\infty} \subset \mathbb{C}$. Then, the sampling formula (10) for $\mathcal{H}_{K}$ can be written as a Lagrange-type interpolation series if and only if the space $\mathcal{H}_{K}$ satisfies the $Z R$ property.

The proof follows the same steps, with only minor changes, as those in [12, Theorem 3.3] for analytic orthogonal Kramer kernels.

\section{Sampling in de Branges spaces}

Paley-Wiener spaces can be seen as special cases of a more general theory of Hilbert spaces of entire functions due to de Branges. We start this section by giving some preliminaries on de Branges spaces needed in the sequel [13, p. 50] (see also [14]). For an entire function $f$ define the function $f^{*}$ as

$$
f^{*}(z):=\overline{f(\bar{z})}, \quad z \in \mathbb{C} .
$$

Definition 3.1 Let $E$ be an entire function verifying $|E(x-i y)|<|E(x+\mathrm{i} y)|$ for all $y>0$. The de Branges space $\mathcal{H}(E)$ is the set of all entire functions $F$ such that

$$
\|F\|_{E}^{2}:=\int_{-\infty}^{\infty}\left|\frac{F(t)}{E(t)}\right|^{2} \mathrm{~d} t<\infty,
$$

and such that both ratios $F / E$ and $F^{*} / E$, where $F^{*}$ is defined by (13), are of bounded type and of non-positive mean type in the upper half-plane.

The structure function or de Branges function $E$ has no zeros in the upper half plane. A de Branges function $E$ is said to be strict if it has no zeros on the real axis. We require that $F / E$ and $F^{*} / E$ be of bounded type and nonpositive mean type in $\mathbb{C}^{+}$. 
A function is of bounded type if it can be written as a quotient of two bounded analytic functions in $\mathbb{C}^{+}$and it is of nonpositive mean type if it grows no faster than $\mathrm{e}^{\varepsilon y}$ for each $\varepsilon>0$ as $y \rightarrow \infty$ on the positive imaginary axis $\{\mathrm{i} y: y>0\}$. A de Branges space $\mathcal{H}(E)$ such that $E$ is a strict de Branges function satisfies the ZR property $[13$, p. 52].

Any de Branges function $E$ can be written as $E(z)=A(z)-\mathrm{i} B(z)$, where $A$ and $B$ are entire functions given by

$$
A(z)=\frac{1}{2}\left(E(z)+E^{*}(z)\right), \quad B(z)=\frac{1}{2 \mathrm{i}}\left(E(z)-E^{*}(z)\right) .
$$

The functions $A$ and $B$ have only real zeros, and these zeros interlace. If $E$ has no real zeros, then all the zeros are simple zeros.

The Paley-Wiener space $P W_{\pi \sigma}$ corresponds to the de Branges space $\mathcal{H}\left(E_{\sigma}\right)$ where $E_{\sigma}(z)=\exp (-\mathrm{i} \pi \sigma z)$.

A de Branges space $\mathcal{H}(E)$ is an RKHS with inner product

$$
\langle f, g\rangle_{E}=\int_{-\infty}^{\infty} \frac{f(t) \overline{g(t)}}{|E(t)|^{2}} \mathrm{~d} t .
$$

Its reproducing kernel

$$
k(w, z)=\frac{B(z) \overline{A(w)}-A(z) \overline{B(w)}}{\pi(z-\bar{w})}, \quad z, w \in \mathbb{C}
$$

has the property that for each $f \in \mathcal{H}(E)$, there holds $f(w)=\langle f, k(w, \cdot)\rangle_{E}$ for all $w \in \mathbb{C}$.

\subsection{Sampling in a de Branges space $\mathcal{H}(E)$}

The existence of a sequence $\left\{t_{n}\right\}$ in $\mathbb{C}$ such that the corresponding sequence $\left\{k\left(t_{n}, \cdot\right)\right\}$ forms an orthogonal basis for $\mathcal{H}(E)$ implies a sampling formula in this space. Namely, any function $f \in \mathcal{H}(E)$ can be recovered from its samples $\left\{f\left(t_{n}\right)\right\}$ through the sampling formula

$$
f(z)=\sum_{n} f\left(t_{n}\right) \frac{k\left(t_{n}, z\right)}{k\left(t_{n}, t_{n}\right)}, \quad z \in \mathbb{C} .
$$

For a proof, expand $f \in \mathcal{H}(E)$ with respect to the orthonormal basis $\left\{k\left(t_{n}, \cdot\right) / \sqrt{k\left(t_{n}, t_{n}\right)}\right\}$ obtaining

$$
f=\sum_{n}\left\langle f, k\left(t_{n}, \cdot\right)\right\rangle_{E} \frac{k\left(t_{n}, \cdot\right)}{k\left(t_{n}, t_{n}\right)}=\sum_{n} f\left(t_{n}\right) \frac{k\left(t_{n}, \cdot\right)}{k\left(t_{n}, t_{n}\right)} \quad \text { in } \mathcal{H}(E) .
$$

The convergence is also absolute and uniform on compact subsets of $\mathbb{C}$.

Following de Branges book [13] there exist sequences $\left\{t_{n}\right\}$ in $\mathbb{R}$ with the above property. Indeed, let $\varphi$ be a phase function associated with $E$, i.e. a continuous function $\varphi(x)$ of real $x$ such that $E(x) \mathrm{e}^{\mathrm{i} \varphi(x)}$ is real-valued for all $x \in \mathbb{R}[13, \mathrm{p} .54]$, and let $\alpha$ be a real number such that the function $\mathrm{e}^{\mathrm{i} \alpha} E(z)-\mathrm{e}^{-i \alpha} E^{*}(z)$ does not belong to $\mathcal{H}(E)$ (there is at most one real number $\alpha \bmod \pi$, such that $\mathrm{e}^{i \alpha} E(z)-\mathrm{e}^{-i \alpha} E^{*}(z) \in \mathcal{H}(E)$ $\left[13\right.$, p. 54]). According to Theorem 22 in [13, p. 55], the sequence of real numbers $\left\{t_{n}\right\}$ satisfying $\varphi\left(t_{n}\right)=\alpha \bmod \pi$ gives the orthogonal basis $\left\{k\left(t_{n}, \cdot\right)\right\}$ for $\mathcal{H}(E)$. 
It is important to mention that the article [15], by Nashed and Walter, is the first reference where sampling in connection with de Branges spaces is introduced. Now we show that the sampling formula (15) is of Lagrange interpolation type.

THEOREM 3.2 There exists an entire function $Q$ having only simple zeros at $\left\{t_{n}\right\}$ such that $\left(z-t_{n}\right) k\left(t_{n}, z\right)=\sigma_{n} Q(z)$ for some nonzero constants $\sigma_{n}$, which allows us to write expansion (15) as the Lagrange-type interpolation formula

$$
f(z)=\sum_{n} f\left(t_{n}\right) \frac{Q(z)}{\left(z-t_{n}\right) Q^{\prime}\left(t_{n}\right)}, \quad z \in \mathbb{C} .
$$

Proof Without loss of generality we can assume that the function $E$ is strict, i.e. it has no real zero. We prove that the only zeros of the function $R_{n}:=k\left(t_{n}, \cdot\right)$ are given by $\left\{t_{r}\right\}_{r \neq n}$. Suppose that $R_{n}(w)=0$; since the space $\mathcal{H}(E)$ satisfies the ZR property $\left[13\right.$, p. 52] we have that $R_{n}(z) /(z-w)$ is in $\mathcal{H}(E)$. Hence, the functions $R_{n}$

$$
\frac{z-t_{n}}{z-w} R_{n}(z)=R_{n}(z)+\frac{w-t_{n}}{z-w} R_{n}(z), \quad z \in \mathbb{C},
$$

also belong to $\mathcal{H}(E)$. If $w \notin\left\{t_{r}\right\}_{r \neq n}$, the analytic function $z \mapsto \frac{z-t_{n}}{z-w} R_{n}(z)$ vanishes at the sequence $\left\{t_{r}\right\}$ which implies that $R_{n} \equiv 0$, to give a contradiction. In addition, the zeros of $R_{n}$ are simple. Indeed, supposing that $t_{m}$ is a multiple zero of $R_{n}$ and proceeding as above, we get that the function $z \mapsto \frac{z-t_{n}}{z-t_{m}} R_{n}(z)$ belongs to $\mathcal{H}(E)$ and vanishes at $\left\{t_{r}\right\}$ which again implies that $R_{n} \equiv 0$.

Consequently, choose an entire function $P$ having only simple zeros at $\left\{t_{n}\right\}$, then for each $n$ there exists an entire function $A_{n}$ without zeros such that $\left(z-t_{n}\right) R_{n}(z)=P(z) A_{n}(z), z \in \mathbb{C}$. Next, we prove that there exists an entire function without zeros $A$ and a sequence $\left\{\sigma_{n}\right\}$ in $\mathbb{C} \backslash\{0\}$ such that $A_{n}(z)=\sigma_{n} A(z)$ for all $z \in \mathbb{C}$. For $m \neq n$ the function $z \mapsto \frac{z-t_{n}}{z-t_{m}} R_{n}(z)$ in $\mathcal{H}(E)$ has its zeros at $\left\{t_{r}\right\}_{r \neq m}$. Thus the sampling formula (15) gives

$$
\frac{z-t_{n}}{z-t_{m}} R_{n}(z)=\left[\left(t_{m}-t_{n}\right) R_{n}^{\prime}\left(t_{m}\right)\right] \frac{R_{m}(z)}{k\left(t_{m}, t_{m}\right)}, \quad z \in \mathbb{C} .
$$

Fixing $m=1$, we conclude that $A_{n}(z)=\sigma_{n} A(z)$ where $A=A_{1} \quad$ and $\sigma_{n}=\left(t_{1}-t_{n}\right) R_{n}^{\prime}\left(t_{1}\right) / k\left(t_{1}, t_{1}\right) \neq 0$ for $n \in \mathbb{N} \backslash\{1\} \quad$ and $\sigma_{1}=1$. Hence, taking $Q(z):=P(z) A(z)$, we have that $k\left(t_{n}, z\right)=\frac{\sigma_{n} Q(z)}{z-T_{n}}$ for $z \neq t_{n}$ and $k\left(t_{n}, t_{n}\right)=\sigma_{n} Q^{\prime}\left(t_{n}\right)$. Substituting this in (15) we derive the Lagrange-type interpolation series (16).

\subsection{Some illustrative example}

Now we connect the sampling results in a de Branges space to a boundary problem involving the Bessel differential equation. For $v \geq-1 / 2$ consider the second order differential Bessel equation:

$$
-u^{\prime \prime}+\left(\frac{v^{2}-\frac{1}{4}}{t^{2}}\right) u=z u, \quad t \in(0,1),
$$

and the boundary condition $u(0)=0$ which is satisfied by the solution $u_{z}(t)=\sqrt{t} J_{v}(t \sqrt{z})$ of (17). Following [16], since $u_{z}(1)=J_{v}(\sqrt{z})$ and $u_{z}^{\prime}(1)=$ $\frac{1}{2} J_{v}(\sqrt{z})+\sqrt{z} J_{v}^{\prime}(\sqrt{z})$ the associated Weyl inner function $\Theta_{v}$ associated with the 
Weyl-Titchmarsh function $m(z)=-\frac{u_{z}^{\prime}(1)}{u_{z}(1)}$ is:

$$
\Theta_{v}(z)=\frac{\sqrt{z} J_{v}^{\prime}(\sqrt{z})+(1 / 2+\mathrm{i}) J_{v}(\sqrt{z})}{\sqrt{z} J_{v}^{\prime}(\sqrt{z})+(1 / 2-\mathrm{i}) J_{v}(\sqrt{z})} .
$$

Given an inner function $\Theta$, we say that a strict de Branges function $E$ is a de Branges function of $\Theta$ if $\Theta=E^{*} / E$ where $E^{*}(z)=\overline{E(\bar{z})}$.

It is well known that $J_{v}(z)=z^{v} G_{v}(z)$ where $G_{v}$ is an even real entire function and $G_{\nu}(0) \neq 0$. The function $F_{\nu}(z)=z G_{v}^{\prime}(z)$ is also an even real entire function. Since $z J_{v}^{\prime}=z^{\nu}\left(v G_{v}+F_{v}\right)$, we can write $(18)$ as

$$
\Theta_{\nu}(z)=\frac{F_{\nu}(\sqrt{z})+(1 / 2+v+\mathrm{i}) G_{\nu}(\sqrt{z})}{F_{\nu}(\sqrt{z})+(1 / 2+v-\mathrm{i}) G_{\nu}(\sqrt{z})} .
$$

The function $E_{v}(z)=F_{v}(\sqrt{z})+(1 / 2+v-i) G_{v}(\sqrt{z})$ is a de Branges function of $\Theta_{v}$. Note that $E_{v}$ does not vanish at $z=0$ and therefore it has no zeros on $\mathbb{R}$. Indeed, $E_{v}(r)=0$ with $r \in \mathbb{R} \backslash\{0\}$ implies that $G_{v}(r)=F_{v}(r)=0$ and, as a consequence, $J_{\nu}(r)=J_{v}(r)=0$.

In what follows, we assume that $v=1 / 2$. In this case $J_{1 / 2}(z)=\sqrt{\frac{2}{\pi z}} \sin z$; hence

$$
G_{1 / 2}(z)=z^{-1 / 2} J_{1 / 2}(z)=\sqrt{\frac{2}{\pi}} \frac{\sin z}{z}, \quad F_{1 / 2}(z)=z G_{1 / 2}^{\prime}(z)=\sqrt{\frac{2}{\pi}} \frac{z \cos z-\sin z}{z} .
$$

Next we deduce some sampling expansions in de Branges space $\mathcal{H}\left(E_{1 / 2}\right)$. Assuming that $E_{1 / 2}(z)=A_{1 / 2}(z)-\mathrm{i} B_{1 / 2}(z)$, in our case,

$$
A_{1 / 2}(z)=F_{1 / 2}(\sqrt{z})+G_{1 / 2}(\sqrt{z}), \quad B_{1 / 2}(z)=G_{1 / 2}(\sqrt{z}) .
$$

A phase function $\phi$ for the space $\mathcal{H}\left(E_{1 / 2}\right)$ is given by

$$
\phi(x)=-\arctan \frac{-G_{1 / 2}(\sqrt{x})}{F_{1 / 2}(\sqrt{x})+G_{1 / 2}(\sqrt{x})} .
$$

For a given real number $\alpha$, the sequence $\left\{r_{n}^{\alpha}\right\}$ should verify

$$
\phi\left(r_{n}^{\alpha}\right)=\alpha \bmod \pi
$$

Thus, for $\alpha=\pi / 2$ the points $r_{n}^{\pi / 2}$ are the zeros of the function $A_{1 / 2}$, and for $\alpha=0$ the points $r_{n}^{0}$ are the zeros of the function $B_{1 / 2}$. For these sequences $\left\{r_{n}^{\alpha}\right\}$ the sequence $\left\{k\left(r_{n}^{\alpha}, z\right)\right\}$ is an orthogonal system for $\mathcal{H}\left(E_{1 / 2}\right)$. Moreover, the sequence $\left\{k\left(r_{n}^{\alpha}, z\right)\right\}$ is an orthogonal basis for $\mathcal{H}\left(E_{1 / 2}\right)$ if and only if the function $\mathrm{e}^{\mathrm{i} \alpha} E(z)-\mathrm{e}^{-\mathrm{i} \alpha} E^{*}(z)$ does not belong to $\mathcal{H}\left(E_{1 / 2}\right)$ [13]. This occurs for $\alpha \in\{0, \pi / 2\}$.

For $\alpha=0$ we have that $\mathrm{e}^{\mathrm{i} \alpha} E_{1 / 2}(z)-\mathrm{e}^{-\mathrm{i} \alpha} E_{1 / 2}^{*}(z)=E_{1 / 2}(z)-E_{1 / 2}^{*}(z)=2 \mathrm{i} B_{1 / 2}(z)$ so we need to check that $B_{1 / 2} \notin \mathcal{H}\left(E_{1 / 2}\right)$. To this end, we check that the function $B_{1 / 2} / E_{1 / 2}$ does not belong to $\mathcal{L}^{2}(\mathbb{R})$. For $x \in \mathbb{R}$, one gets

$$
\left|\frac{B_{1 / 2}(x)}{E_{1 / 2}(x)}\right|^{2}=\frac{B_{1 / 2}^{2}(x)}{A_{1 / 2}^{2}(x)+B_{1 / 2}^{2}(x)}=\frac{\sin ^{2}(\sqrt{x})}{x \cos ^{2}(\sqrt{x})+\sin ^{2}(\sqrt{x})} \notin \mathcal{L}^{1}(\mathbb{R}) .
$$

Having in mind that the points $\left\{r_{n}^{0}\right\}$ are the zeros of the function $B_{1 / 2}(z)=G_{1 / 2}(\sqrt{z})$, that is, $r_{n}^{0}=(n \pi)^{2}$ with $n=1,2, \ldots$, for each $f \in \mathcal{H}\left(E_{1 / 2}\right)$ the 
following sampling formula holds:

$$
f(z)=\sum_{n=1}^{\infty} f\left(n^{2} \pi^{2}\right) \frac{k\left(n^{2} \pi^{2}, z\right)}{k\left(n^{2} \pi^{2}, n^{2} \pi^{2}\right)}, \quad z \in \mathbb{C} .
$$

Having in mind (14) we have

$$
\frac{k\left(n^{2} \pi^{2}, z\right)}{k\left(n^{2} \pi^{2}, n^{2} \pi^{2}\right)}=\frac{2(-1)^{n} n^{2} \pi^{2} \sin (\sqrt{z})}{\left(z-n^{2} \pi^{2}\right) \sqrt{z}} .
$$

The sampling expansion (20) can be written as the Lagrange-type interpolation series

$$
f(z)=\sum_{n=1}^{\infty} f\left(n^{2} \pi^{2}\right) \frac{Q(z)}{\left(z-n^{2} \pi^{2}\right) Q^{\prime}\left(n^{2} \pi^{2}\right)}, \quad z \in \mathbb{C},
$$

where $Q(z)=\sin \sqrt{z} / \sqrt{z}$ for $z \in \mathbb{C}$.

For $\alpha=\pi / 2$ the points of the sequence $\left\{r_{n}^{\pi / 2}\right\}$ are the zeros of the function $A_{1 / 2}$, that is, they are the solutions of

$$
A_{1 / 2}(z)=F_{1 / 2}(\sqrt{z})+G_{1 / 2}(\sqrt{z})=\sqrt{\frac{2}{\pi}} \cos (\sqrt{z})=0 .
$$

Hence, $\quad r_{n}^{\pi / 2}=(2 n-1)^{2} \frac{\pi^{2}}{4}$ with $n=1,2, \ldots$ In this case, the function $\mathrm{e}^{\mathrm{i} \alpha} E_{1 / 2}(z)-\mathrm{e}^{-\mathrm{i} \alpha} E_{1 / 2}^{*}(z) \quad$ equals $\quad \mathrm{i}\left(E_{1 / 2}(z)+E_{1 / 2}^{*}(z)\right)=2 \mathrm{i} A_{1 / 2}(z) . \quad$ As before, $A_{1 / 2} \notin \mathcal{H}\left(E_{1 / 2}\right)$ because the function $A_{1 / 2} / E_{1 / 2}$ does not belong to $\mathcal{L}^{2}(\mathbb{R})$. Indeed,

$$
\left|\frac{A_{1 / 2}(x)}{E_{1 / 2}(x)}\right|^{2}=\frac{A_{1 / 2}^{2}(x)}{A_{1 / 2}^{2}(x)+B_{1 / 2}^{2}(x)}=\frac{x \cos ^{2}(\sqrt{x})}{x \cos ^{2}(\sqrt{x})+\sin ^{2}(\sqrt{x})} \notin \mathcal{L}^{1}(\mathbb{R}) .
$$

Thus, any function $f \in \mathcal{H}\left(E_{1 / 2}\right)$ can be expanded as

$$
\begin{aligned}
f(z) & =\sum_{n=1}^{\infty} f\left([(2 n-1) \pi]^{2} / 4\right) \frac{k\left(\left(\frac{2 n-1}{2}\right)^{2} \pi^{2}, z\right)}{k\left(\left(\frac{2 n-1}{2}\right)^{2} \pi^{2},\left(\frac{2 n-1}{2}\right)^{2} \pi^{2}\right)} \\
& =\sum_{n=1}^{\infty} f\left([(2 n-1) \pi]^{2} / 4\right) \frac{Q(z)}{\left(z-[(2 n-1) \pi]^{2} / 4\right) Q^{\prime}\left([(2 n-1) \pi]^{2} / 4\right)}, \quad z \in \mathbb{C},
\end{aligned}
$$

where $Q(z)=\cos \sqrt{z}$ for $z \in \mathbb{C}$.

Any de Branges space $\mathcal{H}(E)$ can be seen as a $\mathcal{H}_{K}$ space (see Corollary 3.8 below) where $K$ is an analytic Kramer kernel. But this kernel $K$ is not unique. For instance, for the space $\mathcal{H}\left(E_{1 / 2}\right)$ above consider the kernel $K$ defined by

$$
[K(z)](t)=\sqrt{\frac{2}{\pi}} \frac{\sin t \sqrt{z}}{\sqrt{z}}, \quad t \in[0,1], \quad z \in \mathbb{C} .
$$

For any $z \in \mathbb{C}$ the function $K(z)$ belongs to $\mathcal{L}^{2}[0,1]$ and the map $\mathbb{C} \ni z \mapsto K(z) \in \mathcal{L}^{2}[0,1]$ is analytic. Moreover, the anti-linear map

$$
\mathcal{L}^{2}[0,1] \rightarrow \mathcal{H}\left(E_{1 / 2}\right), \quad f \mapsto \int_{0}^{1}[K(z)](t) \overline{f(t)} \mathrm{d} t
$$


is an isometric map [16]. The challenging problem is to determine when a $\mathcal{H}_{K}$ space is equal isometrically to a de Branges space.

\subsection{A new characterization of de Branges spaces}

Consider $\mathcal{H}$ an RKHS of entire functions on $\mathbb{C}$ with reproducing kernel $k$. In this section we give a new characterization for $\mathcal{H}$ being a de Branges space. To this end, we use the following classical characterization of a de Branges space which can be found in [13, p. 57]:

THEOREM 3.3 A Hilbert space $\mathcal{H}$ of entire functions is equal isometrically to some de Branges space $\mathcal{H}(E)$ if and only if the following conditions hold:

B1. Whenever $f \in \mathcal{H}$ and $\omega$ is a nonreal zero of $f$, the function

$$
g(z):=\frac{z-\bar{\omega}}{z-\omega} f(z)
$$

belongs to $\mathcal{H}$ and $\|g\|=\|f\|$.

B2. For each $\omega \notin \mathbb{R}$ the linear mapping $\mathcal{H} \ni f \rightarrow f(\omega) \in \mathbb{C}$ is continuous.

B3. The function $f^{*}$ defined as in (13) belongs to the space, and $\left\|f^{*}\right\|=\|f\|$.

Now, suppose $\mathcal{H}$ is a Hilbert space of entire functions on $\mathbb{C}$ and $k$ is its reproducing kernel. The following is a rather minor variation of Theorem $B$ in [17] as well as of the reproducing Hilbert space test identified as $(\eta)$ in [18].

Lemma 3.4 Suppose that $\Omega$ is a set of uniqueness of $\mathcal{H}$. A complex function $f$ is in $\mathcal{H}$ if and only if there exist $C>0$ such that

$$
\left|\sum_{i=0}^{N} \lambda_{i} f\left(z_{i}\right)\right|^{2} \leq C^{2} \sum_{i, j=0}^{N} \lambda_{i} \bar{\lambda}_{j} k\left(z_{i}, z_{j}\right), \quad z_{0}, \ldots, z_{N} \in \Omega, \lambda_{0}, \ldots \lambda_{N} \in \mathbb{C} .
$$

A couple of words for the proof: Due to (21), the map

$$
\Phi: \sum_{i=0}^{N} \lambda_{i} f\left(z_{i}\right) \rightarrow \sum_{i=0}^{N} \lambda_{i} k_{z_{i}}
$$

defines a bounded linear functional on the closure of $\left\{k_{z}: z \in \Omega\right\}$; extend it by putting 0 on the orthogonal complement of its (temporarily) domain. Consequently, by the Riesz representation theorem there is $\tilde{f}$ in $\mathcal{H}$, which, by the reproducing kernel property, agrees with $f$ on $\Omega$. Therefore $\widetilde{f}$ coincides with $f$.

For $\omega \in \mathbb{C}$ denote by $\mathcal{H}_{\omega}$ all the functions $f$ in $\mathcal{H}$ such that $f(\omega)=0$. Furthermore, define for $f \in \mathcal{H}_{\omega}$ the function $f_{\omega}$ by $f_{\omega}:=(z-\bar{\omega})(z-\omega)^{-1} f(z)$

THEOREM $3.5 \mathcal{H}$ is a de Branges space if and only if it is isometrically imbedded into $\mathcal{L}^{2}(\mu)$ with some positive measure on $\mathbb{R}$ and satisfies the following approximation condition:

For any $\omega \in \mathbb{C}, f \in \mathcal{H}_{\omega}$ and any finite subset $C \subset \mathbb{C}$ there is a sequence $\left(\varphi_{C, n}\right)_{n} \subset \mathcal{H}$ such that $\varphi_{C, n}(z) \rightarrow f_{\omega}(z), z \in C$, and the norms

$\left\|\varphi_{C, n}\right\|_{\mathcal{L}^{2}(\mu)}<M$ with $M$ depending on $f$ exclusively,

and $\mathcal{H}$ is symmetric with respect to the involution $f \rightarrow f^{*}$. 
Proof Prove first that if $f$ belongs to some $\mathcal{H}_{\omega}$, then the function $f_{\omega}$ belongs to $\mathcal{H}$ and $\left\|f_{\omega}\right\|=\|f\|$. For the latter note that

$$
\int_{\mathbb{R}}\left|f_{\omega}(z)\right|^{2} \mu(\mathrm{d} z)=\int_{\mathbb{R}}\left|(z-\bar{\omega})(z-\omega)^{-1} f(z)\right|^{2} \mu(\mathrm{d} z)=\int_{\mathbb{R}}|f(z)|^{2} \mu(\mathrm{d} z)
$$

because $|(z-\bar{\omega})|=|(z-\omega)|$ as long as $z$ is real. This means in particular that $f_{\omega}$ is in $\mathcal{L}^{2}(\mu)$.

Suppose now that $f$ belongs to some $\mathcal{H}_{\omega}$. Choose a finite set $\left(z_{i}\right)_{i=0}^{N}$ such that $C:=\left(z_{i}\right)_{i=0}^{N} \subset \Omega:=\mathbb{C} \backslash\{\omega\}$ and $\left(\lambda_{i}\right)_{i=0}^{N} \subset \mathbb{C}$. Let $\left(\varphi_{C, n}\right)_{n}$ be the sequence appearing in (22). Fix $\epsilon>0$ such that $\left|\sum_{i=0}^{N} \lambda_{i}\left(f_{\omega}\left(z_{i}\right)-\varphi_{C, n}\left(z_{i}\right)\right)\right|<\epsilon$ for $n$ sufficiently large ( $C$ is finite).

Using the reproducing kernel property on the way we can write

$$
\begin{aligned}
\left|\sum_{i=0}^{N} \lambda_{i} f_{\omega}\left(z_{i}\right)\right| & =\left|\sum_{i=0}^{N} \lambda_{i} \varphi_{C, n}\left(z_{i}\right)\right|+\left|\sum_{i=0}^{N} \lambda_{i}\left(f_{\omega}\left(z_{i}\right)-\varphi_{C, n}\left(z_{i}\right)\right)\right| \\
& \leq\left|\left\langle\varphi_{C, n}, \sum_{i} \lambda_{i} k_{z_{i}}\right\rangle_{\mathcal{H}}\right|+\epsilon \leq\left\|\varphi_{C, n}\right\|_{\mathcal{L}^{2}(\mu)}\left\|\sum_{i} \lambda_{i} k_{z_{i}}\right\|_{\mathcal{L}^{2}(\mu)}+\epsilon \\
& =M\left\|\sum_{i} \lambda_{i} k_{z_{i}}\right\|_{\mathcal{H}}^{2}+\epsilon=M\left(\sum_{i, j} \lambda_{i} \bar{\lambda}_{j} k\left(z_{i}, z_{j}\right)\right)^{\frac{1}{2}}+\epsilon .
\end{aligned}
$$

Removing $\epsilon$ we come to (21). Because $\Omega$ is a set of uniqueness for $\mathcal{H}$, Lemma 3.4 applies to conclude that $f_{\omega}$ is in $\mathcal{H}$ as well.

The involution $f \rightarrow f^{*}$ is an isometry in $\mathcal{L}^{2}(\mu)$. Because the involution $f \rightarrow f^{*}$ is symmetric the second condition needed is satisfied as well. The converse implication required in the theorem is transparent.

As a consequence of Theorem 3.5 we give the following example.

\subsubsection{A supporting example}

Suppose that $\left(p_{n}\right)_{n=0}^{\infty}$ is a sequence of real polynomials orthonormal with respect to an indeterminate measure $\mu$. Then [19, Theorem 10.33],

$$
K(z, w):=\sum_{n=0}^{\infty} p_{n}(z) \overline{p_{n}(w)}, \quad z, w \in \mathbb{C},
$$

is well defined. For $K_{N}(z, w):=\sum_{n=0}^{N} p_{n}(z) \overline{p_{n}(w)}$ to be a finite truncation of the kernel $K$ we have

$$
\begin{aligned}
\int_{\mathbb{R}} K_{N}(z, t) \overline{K_{N}(w, t)} \mu(\mathrm{d} t) & =\sum_{m, n=0}^{N} p_{m}(z) \overline{p_{n}(w)} \int_{\mathbb{R}} \overline{p_{m}(t)} p_{n}(t) \mu(\mathrm{d} t) \\
& =\sum_{n=0}^{N} p_{n}(z) \overline{p_{n}(w)}=K_{N}(z, w) .
\end{aligned}
$$


Therefore (cf. [20, Theorem 1.38, p. 28]), we have

$$
\begin{aligned}
\int_{\mathbb{R}} K(z, t) \overline{K(w, t)} \mu(\mathrm{d} t) & =\lim _{N \rightarrow+\infty} \int_{\mathbb{R}} K_{N}(z, t) \overline{K_{N}(w, t)} \mu(\mathrm{d} t)=K(z, w) \\
& =\langle K(\cdot, w), K(\cdot, z)\rangle
\end{aligned}
$$

and, consequently, the Hilbert space $\mathcal{H}$ corresponding to the kernel is isometrically imbedded in $\mathcal{L}^{2}(\mu)$.

Because the polynomials $p_{n}$ are of real coefficients, $\mathcal{H}$ is symmetric with respect to the involution $f \rightarrow f^{*}$.

Because the polynomials $\left(p_{n}\right)_{n}$ form an orthonormal basis of $\mathcal{H}$ (cf. Fact $\mathrm{B}$ in [18]) all the polynomials are in $\mathcal{H}$. Furthermore, because $\mathcal{H}_{\omega}$ is closed in $\mathcal{H}$ (sic!), for any $f \in \mathcal{H}_{\omega}$ there is a sequence $\left(r_{n}\right)_{n}$ of polynomials in $\mathcal{H}_{\omega}$ converging to $f$ in the norm of $\mathcal{H}$ and such that $\left\|r_{n}\right\|_{\mathcal{L}^{2}(\mu)}=\left\|r_{n}\right\|_{\mathcal{H}} \leq 2\|f\|_{\mathcal{H}}$, say. Therefore, $\left(r_{n}\right)_{n}$ plays the role of $\left(\varphi_{C, n}\right)_{n}$ in the approximation property (22) (note that $C$ becomes irrelevant as norm convergence in $\mathcal{H}$ implies that of uniform one on compact subsets of $\mathbb{C}$ ). Because the polynomials $r_{n}$ 's are in $\mathcal{H}_{\omega}$, according to $(23),\left(r_{n}\right)_{\omega}$ 's are still polynomials, of the same norm as that of $r_{n}$ 's. Now we are in a position to apply Theorem 3.5 and conclude with the following corollary:

COROLlary 3.6 The above constructed $\mathcal{H}$ is a de Branges space.

The same refers to polynomials of the second kind [19, Theorem 10.33].

\subsection{The spaces $\mathcal{H}_{K}$ as de Branges spaces}

In this section we are interested in the search of a sampling characterization of the spaces $\mathcal{H}_{K}$ as de Branges spaces. Whenever the RKHS $\mathcal{H}_{K}$ associated with a Kramer kernel $K$ is (equal isometrically to) a de Branges space $\mathcal{H}(E)$ such that the entire function $E$ has no real zeros, then the space $\mathcal{H}_{K}$ satisfies the ZR property [13, p. 52]. As a consequence, the sampling formula (10) in $\mathcal{H}_{K}$ can be written as a Lagrange-type interpolation formula. There exists a form of a converse result in the case that the sequence $\left\{z_{n}\right\}_{n=1}^{\infty}$ is real, and the function $P$ is real for real $z$.

THEOREM 3.7 Assume that the sampling formula (10) is an orthogonal expansion in $\mathcal{H}_{K}$ and that it can be written as a Lagrange-type interpolation formula (2.5) where the sampling points $\left\{t_{n}\right\}_{n=1}^{\infty}$ are real, and $P$ is an entire function having simple zeros at $\left\{t_{n}\right\}_{n=1}^{\infty}$ and satisfying $P^{*}=P$. Then, the space $\mathcal{H}_{K}$ is a de Branges space.

Proof In the present case, property B2 holds because $\mathcal{H}_{K}$ is a RKHS. For B3, consider $f \in \mathcal{H}_{K}$ such that $f(z)=\langle K(z), x\rangle$ for some $x \in \mathcal{H}$. Then, $f^{*}(z)=\overline{\langle K(\bar{z}), x\rangle}=\sum_{n=1}^{\infty} S_{n}^{*}(z)\left\langle x, e_{n}\right\rangle$, where $\left\{e_{n}\right\}_{n=1}^{\infty}$ denotes an orthonormal basis such that $K\left(t_{n}\right)=a_{n} e_{n}$ for all $n \in \mathbb{N}$. From (11) we find that $\left\langle x, e_{n}\right\rangle=f^{*}\left(t_{n}\right) / \bar{a}_{n}, n \in \mathbb{N}$. Since

$$
S_{n}(z)=a_{n} \frac{P(z)}{\left(z-t_{n}\right) P^{\prime}\left(t_{n}\right)}, \quad n \in \mathbb{N},
$$

$P^{*}=P$ and $\left\{t_{n}\right\}_{n=1}^{\infty} \subset \mathbb{R}$, we obtain

$$
S_{n}^{*}(z)=\bar{a}_{n} \frac{P(z)}{\left(z-t_{n}\right) P^{\prime}\left(t_{n}\right)}, \quad n \in \mathbb{N} .
$$


Thus we get

$$
f^{*}(z)=\sum_{n=1}^{\infty} f^{*}\left(t_{n}\right) \frac{P(z)}{\left(z-t_{n}\right) P^{\prime}\left(t_{n}\right)} .
$$

Let $y$ be in $\mathcal{H}$ such that its Fourier coefficients with respect to the orthonormal basis $\left\{e_{n}\right\}_{n=1}^{\infty}$ are

$$
\left\langle y, e_{n}\right\rangle=\frac{a_{n}}{\bar{a}_{n}} \overline{\left\langle x, e_{n}\right\rangle}, \quad n \in \mathbb{N} .
$$

The function $g(z):=\langle K(z), y\rangle$ in $\mathcal{H}_{K}$ satisfies $g\left(t_{n}\right)=a_{n} \overline{\left\langle y, e_{n}\right\rangle}=\bar{a}_{n}\left\langle x, e_{n}\right\rangle=f^{*}\left(t_{n}\right)$ for each $n \in \mathbb{N}$. Taking into account (24) we conclude that $f^{*}=g$ and, as a consequence, $f^{*} \in \mathcal{H}_{K}$. Moreover,

$$
\left\|f^{*}\right\|^{2}=\|y\|_{\mathcal{H}}^{2}=\sum_{n=1}^{\infty}\left|\left\langle y, e_{n}\right\rangle\right|^{2}=\sum_{n=1}^{\infty}\left|\left\langle x, e_{n}\right\rangle\right|^{2}=\|x\|_{\mathcal{H}}^{2}=\|f\|^{2} .
$$

Finally we prove property $\mathrm{B} 1$. To this end, consider $f \in \mathcal{H}_{K}$ given by $f(z)=\langle K(z), x\rangle$ for some $x \in \mathcal{H}$, and such that $f(w)=0$ where $w \in \mathbb{C} \backslash \mathbb{R}$. Since $P(w) \neq 0$, the Lagrange-type interpolation formula for $f$ gives

$$
\sum_{n=1}^{\infty} \frac{f\left(t_{n}\right)}{\left(w-t_{n}\right) P^{\prime}\left(t_{n}\right)}=0
$$

Therefore,

$$
\begin{aligned}
f(z) & =\sum_{n=1}^{\infty} f\left(t_{n}\right) \frac{P(z)}{\left(z-t_{n}\right) P^{\prime}\left(t_{n}\right)}-\sum_{n=1}^{\infty} f\left(t_{n}\right) \frac{P(z)}{\left(w-t_{n}\right) P^{\prime}\left(t_{n}\right)} \\
& =(w-z) \sum_{n=1}^{\infty} f\left(t_{n}\right) \frac{P(z)}{P^{\prime}\left(t_{n}\right)} \frac{1}{\left(z-t_{n}\right)\left(w-t_{n}\right)} .
\end{aligned}
$$

As a consequence, we obtain

$$
\frac{f(z)}{z-w}=\sum_{n=1}^{\infty} \frac{f\left(t_{n}\right)}{\left(t_{n}-w\right)} \frac{P(z)}{\left(z-t_{n}\right) P^{\prime}\left(t_{n}\right)}
$$

Since

$$
\frac{z-\bar{w}}{z-w} f(z)=f(z)+(w-\bar{w}) \frac{f(z)}{z-w}
$$

the function $[(z-\bar{w}) /(z-w)] f(z)$ belongs to $\mathcal{H}_{K}$ if and only if the function $f(z) /(z-w)$ belongs to $\mathcal{H}_{K}$ which follows from Theorem 2.5. As in the proof of Theorem 2.5, the function $g \in \mathcal{H}_{K}$ defined by $g(z):=\langle K(z), y\rangle$, where $y \in \mathcal{H}$ has Fourier coefficients with respect to the orthonormal basis $\left\{e_{n}\right\}_{n=1}^{\infty}$

$$
\left\langle y, e_{n}\right\rangle=\frac{1}{t_{n}-\bar{w}}\left\langle x, e_{n}\right\rangle, \quad n \in \mathbb{N},
$$


coincides with the entire function $f(z) /(z-w)$. Moreover,

$$
\begin{aligned}
\left\|\frac{z-\bar{w}}{z-w} f(z)\right\|^{2} & =\|f+(w-\bar{w}) g\|^{2}=\|x+(\bar{w}-w) y\|_{\mathcal{H}}^{2} \\
& =\sum_{n=1}^{\infty}\left|\left\langle x+(\bar{w}-w) y, e_{n}\right\rangle\right|^{2}=\sum_{n=1}^{\infty}\left|\frac{t_{n}-w}{t_{n}-\bar{w}}\right|^{2}\left|\left\langle x, e_{n}\right\rangle\right|^{2}=\|x\|_{\mathcal{H}}^{2}=\|f\|^{2},
\end{aligned}
$$

which concludes the proof.

As a consequence of Theorem 3.7, we find the following characterization of the de Branges spaces as $\mathcal{H}_{K}$ spaces.

COROLlary 3.8 A space $\mathcal{H}_{K}$ is a de Branges space (that means, equal isometrically to some de Branges space $\mathcal{H}(E)$ ) if and only if there exists an orthogonal sampling formula in $\mathcal{H}_{K}$ such that it can be written as a Lagrange-type interpolation formula, i.e. for each $f \in \mathcal{H}_{K}$,

$$
f(z)=\sum_{n} f\left(t_{n}\right) \frac{P(z)}{\left(z-t_{n}\right) P^{\prime}\left(t_{n}\right)}, \quad z \in \mathbb{C},
$$

where $\left\{t_{n}\right\}_{n=1}^{\infty}$ is a real sequence of sampling points and $P^{*}(z)=P(z)$ for all $z \in \mathbb{C}$.

Proof To conclude the result we should identify the de Branges space $\mathcal{H}(E)$ with a $\mathcal{H}_{K}$ space. Indeed, for any $f \in \mathcal{H}(E)$ we have

$$
f(z)=\langle f(w), k(z, w)\rangle_{E}=\int_{\mathbb{R}} f(t) \frac{\overline{k(z, t)}}{|E(t)|^{2}} \mathrm{~d} t=\left\langle K(z), f^{*}\right\rangle_{E}, \quad z \in \mathbb{C} .
$$

where the Kramer analytic kernel is given by $[K(z)](w)=\frac{B(w) A(z)-A(w) B(z)}{\pi(w-z)}$. Note that the functions $f^{*}$ and $K(z), z \in \mathbb{C}$, belong to $\mathcal{H}(E)$.

\subsubsection{The example in 3.3.1 revisited}

Throughout this section we follow the reference [6] and the references therein. Let $s=\left\{s_{n}\right\}_{n \in \mathbb{N}_{0}}$ be an indeterminate Hamburger moment sequence and let $V_{s}$ be the set of positive Borel measures $\mu$ on $\mathbb{R}$ satisfying $\int_{-\infty}^{\infty} x^{n} \mathrm{~d} \mu(x)=s_{n}, n \in \mathbb{N}_{0}:=\mathbb{N} \cup\{0\}$. The functional $\mathcal{L}$ defined on the vector space $\mathbb{C}[x]$ of polynomials $p(x)=\sum_{k=0}^{n} p_{k} x^{k}$ by $\mathcal{L}(p)=\sum_{k=0}^{n} p_{k} s_{k}=\int_{-\infty}^{\infty} p(x) \mathrm{d} \mu(x)$ is independent of $\mu \in V_{s}$. Let $\left\{P_{n}\right\}_{n \in \mathbb{N}_{0}}$ be the corresponding orthonormal polynomials satisfying

$$
\int_{-\infty}^{\infty} P_{n}(x) P_{m}(x) \mathrm{d} \mu(x)=\delta_{n m}, \quad \text { for each } \mu \in V_{s} .
$$

We assume that $P_{n}$ is of degree $n$ with positive leading coefficient. The set $V_{s}$ of solutions to an indeterminate moment problem can be parametrized with the one-point compactification $\mathcal{P} \cup\{\infty\}$ of the Pick (or Herglotz) functions set $\mathcal{P}$ [21]. When the parameter is restricted to constant functions taking values in $\mathbb{R} \cup\{\infty\}$, we obtain the set of $\mathrm{N}$-extremal measures $\left\{\mu_{t}\right\}$ which satisfy

$$
\int_{-\infty}^{\infty} \frac{\mathrm{d} \mu_{t}(x)}{x-z}=-\frac{A(z) t-C(z)}{B(z) t-D(z)}, \quad z \in \mathbb{C} \backslash \mathbb{R},
$$


where $A, B, C, D$ are the entire functions forming the so-called Nevanlinna matrix associated with the moment problem. See [21] for explicit formulas of the Nevanlinna matrix. It is known that, for each $t \in \mathbb{R} \cup\{\infty\}, \mu_{t}$ is the discrete measure $\mu_{t}=\sum_{z \in \Lambda_{t}} m_{z} \delta_{z}$ where

$$
\Lambda_{t}= \begin{cases}\{z \in \mathbb{C} \mid B(z) t-D(z)=0\} & \text { if } t \in \mathbb{R}, \\ \{z \in \mathbb{C} \mid B(z)=0\} & \text { if } t=\infty,\end{cases}
$$

and

$$
m_{z}=\frac{A(z) t-C(z)}{B^{\prime}(z) t-D^{\prime}(z)}, \quad \text { for } z \in \Lambda_{t}
$$

Recall that the zeros of the entire function $B(z) t-D(z)$ or $B(z)$ are real and simple, and they form a sequence $\left\{z_{m}^{t}\right\}_{m=0}^{\infty}$. The $\mathrm{N}$-extremal measures are characterized as those measures $\mu \in V_{s}$ for which the polynomials are dense in $\mathcal{L}^{2}(\mu)$.

On the other hand, it is known that the sequence in $\ell^{2}\left(\mathbb{N}_{0}\right)$ given by

$$
\left\{P_{0}\left(z_{m}^{t}\right)_{1}\left(z_{m}^{t}\right)_{2}\left(z_{m}^{t}\right)_{3}\left(z_{m}^{t}\right), \ldots .\right\}_{m \in \mathbb{N}_{0}}
$$

is an orthogonal basis for $\ell^{2}\left(\mathbb{N}_{0}\right)$. As a consequence, $[K(z)](n)=P_{n}(z), n \in \mathbb{N}_{0}$ defines an analytic Kramer kernel in $\ell^{2}\left(\mathbb{N}_{0}\right)$. Moreover, the following sampling theorem in the corresponding $\mathcal{H}_{K}$ space holds see [6]:

THEOREM 3.9 Let $\mu_{t}$ be an N-extremal measure for an indeterminate moment problem and let $\left\{P_{n}\right\}_{n=0}^{\infty}$ be the sequence of associated orthonormal polynomials. Assume that $\left\{z_{m}^{t}\right\}_{m=0}^{\infty}$ are the zeros of $B(z) t-D(z)$ if $t \in \mathbb{R}$, or the zeros of $B(z)$ if $t=\infty$. Then, any function $F(z)=\sum_{n=0}^{\infty} c_{n} P_{n}(z)$ where $\left\{c_{n}\right\}_{n=0}^{\infty} \in \ell^{2}\left(\mathbb{N}_{0}\right)$ can be recovered from its samples $\left\{F\left(z_{m}^{t}\right)\right\}_{m=0}^{\infty}$ through the Lagrange-type interpolatory series

$$
F(z)=\sum_{m=0}^{\infty} F\left(z_{m}^{t}\right) \frac{G_{t}(z)}{G_{t}^{\prime}\left(z_{m}^{t}\right)\left(z-z_{m}^{t}\right)},
$$

where

$$
G_{t}(z)= \begin{cases}B(z) t-D(z) & \text { if } t \in \mathbb{R} \\ B(z) & \text { if } t=\infty .\end{cases}
$$

The series (26) converges absolutely and uniformly on compact subsets of $\mathbb{C}$.

As a consequence of Theorem 3.9 and Corollary 3.8 we deduce that the space $\mathcal{H}_{K}:=\left\{f(z)=\sum_{n=0}^{\infty} a_{n} P_{n}(z), z \in \mathbb{C}\right.$ where $\left.\left\{a_{n}\right\}_{n=0}^{\infty} \in \ell^{2}\left(\mathbb{N}_{0}\right)\right\}$, is a de Branges space.

Another good candidate to be an analytic Kramer kernel is $[K(z)](n)=Q_{n}(z)$, $n \in \mathbb{N}$, where $\left\{Q_{n}(z)\right\}_{n=1}^{\infty}$ denotes the sequence of the second kind orthogonal polynomials associated to $\left\{P_{n}\right\}_{n=0}^{\infty}$. These polynomials are given in terms of the measure $\mu_{t}$ by $Q_{n}(z)=\int \frac{P_{n}(z)-P_{n}(u)}{z-u} \mathrm{~d} \mu_{t}(u)$. Any function $f(z)=\sum_{n=1}^{\infty} a_{n} Q_{n}(z)$ can be recovered by means of a Lagrange-type interpolation series as in Theorem 3.9 (see [6]); consequently, $\mathcal{H}_{K}:=\left\{f(z)=\sum_{n=1}^{\infty} a_{n} Q_{n}(z)\right.$ where $\left.\left\{a_{n}\right\}_{n=1}^{\infty} \in \ell^{2}(\mathbb{N})\right\}$ is a de Branges space.

\section{Dedicatory}

Antonio G. García and Miguel A. Hernández-Medina are very pleased to dedicate their work to Prof F.H. Szafraniec on the occasion of his 70 th birthday. The research 
of Prof Szafraniec has inspired and influenced many mathematicians throughout the world; we are fortunate to be two of those.

\section{Acknowledgements}

The work of Antonio G. García and Miguel A. Hernández-Medina has been supported by grant no. MTM2009-08345 from the Spanish Ministerio de Ciencia e Innovación (MICINN). The work of Franciszek Hugon Szafraniec has been supported by grant nos. N N201 364436 and N N201 546438 of the Ministry of Science and Higher Education (MNiSzW), Poland.

\section{Notes}

1. Details are given in the next section.

2. This kind of construction has been appearing for a long time in many diverse circumstances, an extensive assortment of instances can be found in the monograph [22]; for a general setup refer [18].

\section{References}

[1] W.N. Everitt and G. Nasri-Roudsari, Interpolation and sampling theories, and linear ordinary boundary value problems, in Sampling Theory in Fourier and Signal Analysis: Advanced Topics, J.R. Higgins and R.L. Stens, eds., Chapter 5, Oxford University Press, Oxford, 1999, pp. 96-129.

[2] H.P. Kramer, A generalized sampling theorem, J. Math. Phys. 63 (1957), pp. 68-72.

[3] A.I. Zayed, Advances in Shannon's Sampling Theory, CRC Press, Boca Raton, 1993.

[4] P. Butzer, J.R. Higgins, and R.L. Stens, Sampling theory in signal analysis, in Development of Mathematics 1950-2000, J.P. Pier, ed., Birkäuser, Basel, 2000, pp. 193-234.

[5] P. Butzer and G. Nasri-Roudsari, Kramer's sampling theorem in signal analysis and its role in mathematics, in Image Processing; Mathematical Methods and Applications (Proceedings of IMA Conference, Cranfield University, UK), J.M. Blackledge ed., Clarendon Press, Oxford, 1997, pp. 49-95.

[6] A.G. Garcia and M.A. Hernández-Medina, The discrete Kramer sampling theorem and indeterminate moment problems, J. Comp. Appl. Math. 134 (2001), pp. 13-22.

[7] W.N. Everitt, G. Nasri-Roudsari, and J. Rehberg, A note on the analytic form of the Kramer sampling theorem, Results Math. 34(3-4) (1998), pp. 310-319.

[8] R.M. Young, An Introduction to Nonharmonic Fourier Series, Academic Press, New York, 1980.

[9] A.E. Taylor and D.C. Lay, Introduction to Functional Analysis, John Wiley \& Sons, New York, 1980.

[10] J.R. Higgins, A sampling principle associated with Saitoh's fundamental theory of linear transformations, in Analytic Extension Formulas and their Applications, S. Saitoh, N. Nayashi, and M. Yamamoto, eds., Kluwer Academic, Pordrecht, 2001, pp. 73-86.

[11] A.G. García and F.H. Szafraniec, A converse of the Kramer sampling theorem, Sampl. Theory Signal Image Process. 1(1) (2002), pp. 53-61.

[12] W.N. Everitt, A.G. García, and M.A. Hernández-Medina, On Lagrange-type interpolation series and analytic Kramer kernels, Results Math. 51 (2008), pp. 215-228.

[13] L. de Branges, Hilbert Spaces of Entire Functions, Prentice-Hall, Englewood Cliffs, NJ, 1968.

[14] J.C. Lagarias, Hilbert Spaces of Entire Functions and Dirichlet l-functions, Frontiers in Number Theory, Physics, and Geometry I, Springer, Berlin, 2006, pp. 367-378. 
[15] M.Z. Nashed and G.G. Walter, General sampling theorems for functions in reproducing kernel spaces, Math. Control Signals Syst. 4 (1991), pp. 363-390.

[16] N. Makarov and A. Poltoratski, Meromorphic Inner Functions, Toeplitz Kernels, and the Uncertainty Principle, Mathematical Physics Studies, Vol. 27, Springer, Berlin, 2006, pp. $185-252$.

[17] F.H. Szafraniec, Interpolation and domination by positive definite kernels, in Complex Analysis - Fifth Romanian-Finish Seminar, Part 2 (Proceedings, Bucarest Romania, 1981), C. Andrean Cazacu, N. Boboc, M. Jurchescu, and I. Suciu, eds., Lecture Notes in Mathematics, Vol. 1014, Springer, Berlin-Heidelberg, 1983, pp. 291-295.

[18] F.H. Szafraniec, The reproducing kernel Hilbert space and its multiplication operators, Oper. Theory Adv. Appl. 114 (2000), pp. 253-263.

[19] M.H. Stone, Linear Transformations in Hilbert Space, American Mathematical Society Colloquium Publications, Vol. 15, Americal Mathematical Society, Providence, RI, 1932.

[20] W. Rudin, Real and Complex Analysis, McGraw-Hill Publishing Company, New York, 1970.

[21] B. Simon, The classical moment problem as a self-adjoint finite difference operator, Adv. Math. 137 (1998), pp. 82-203.

[22] S. Saitoh, Integral Transforms, Reproducing Kernels and their Applications, Longman, Essex, England, 1997.

[23] A.G. García and L.L. Littlejohn, On analytic sampling theory, J. Comp. Appl. Math. 171 (2004), pp. 235-246. 